these issues from clinicians, patients, and the medical-device industry.

Daniel G. Schultz, M.D.

Food and Drug Administration

Rockville, MD 20850

execsec@fda.hhs.gov

1. Maisel WH. Semper fidelis - consumer protection for patients with implanted medical devices. N Engl J Med 2008;358:985-7.

THE AUTHOR REPLIES: Because of their inherent complexity and widespread distribution, medical devices will occasionally malfunction, fail, or cause unexpected adverse events. It is challenging to predict the long-term performance of implanted devices on the basis of bench testing or premarketing clinical trials of short duration. Thus, for selected life-sustaining products, postmarketing studies of sufficient size and duration must be required of manufacturers. Unfortunately, when questions first arose about the performance of the Medtronic Sprint Fidelis ICD lead, the available postmarketing data were insufficient to allow an accurate evaluation of the device's performance. Over the ensuing months, tens of thousands of additional patients underwent implantation of the lead, only to be notified of its recall a short time later.

There is no "deeper" problem than the unnecessary exposure of patients to an underperforming permanently implanted medical device. Schultz outlines several important questions that must be answered in order to strengthen the device-approval and postmarketing-monitoring processes. In addition, technological advances such as remote, wireless device monitoring will, in some cases, facilitate collection of the needed performance data. More critical, however, is the need for a philosophical shift - from a medicaldevice industry that primarily reacts to performance failures to one that better anticipates them.

William H. Maisel, M.D., M.P.H.

Beth Israel Deaconess Medical Center

Boston, MA 02215

wmaisel@bidmc.harvard.edu

\title{
Mechanical Ventilation and Disuse Atrophy of the Diaphragm
}

TO THE EDITOR: In their article, Levine et al. (March 27 issue) ${ }^{1}$ conclude that atrophy of the human diaphragm occurs with mechanical ventilation; this finding is consistent with our observation of atrophy of the diaphragm in a patient with a high spinal cord injury after 8 months of mechanical ventilation necessitated by the failure of one diaphragmatic pacemaker. ${ }^{2}$ In this patient, atrophy of the diaphragm was prevented in the hemidiaphragm connected to the functioning pacemaker by stimulating the pacemaker for only 30 minutes per day. Levine et al. speculate as to whether there are functional implications due to atrophy and whether strategies can be used to prevent atrophy. Our study addresses both of these questions. First, we found that atrophy was associated with profound reductions in tidal volume. Second, we found that stimulation of the phrenic nerve of the diaphragm for 30 minutes per day was sufficient to prevent atrophy. From our observations and those of Levine et al., one major question arises: Is the diaphragmatic activation associated with the forms of ventilation commonly used in the intensive care unit sufficient to prevent disuse atrophy?

Dennis McCool, M.D.

Brown University

Providence, RI 02860

f_mccool@brown.edu

Najib Ayas, M.D.

University of British Columbia

Vancouver, BC V6T 1Z4, Canada

Robert Brown, M.D.

Massachusetts General Hospital Boston, MA 02114

1. Levine S, Nguyen T, Taylor N, et al. Rapid disuse atrophy of diaphragm fibers in mechanically ventilated humans. $\mathrm{N}$ Engl J Med 2008;358:1327-35.

2. Ayas NT, McCool FD, Gore R, Lieberman SL, Brown R. Prevention of human diaphragm atrophy with short periods of electrical stimulation. Am J Respir Crit Care Med 1999;159:2018-20.

TO THE EDITOR: Levine and colleagues confirm that in the absence of respiratory effort, mechanical ventilation for as little as 1 day causes profound atrophy of the diaphragm in adult humans, as it does in animals. Two decades ago, Knisely 
and others ${ }^{1}$ reported dramatic evidence of ventilation-induced atrophy in infants. They compared autopsy material obtained from 13 infants who died after mechanical ventilation for at least 12 days with that obtained from 26 infants who died after ventilation for 7 or days or less. Infants who were ventilated for a longer time had smaller diaphragm myofibers with histologic features of disuse atrophy, whereas strap and tongue muscles appeared to be normal. ${ }^{2}$ To control for the nutritional and catabolic effects of illness, they assessed ratios of the cross-sectional areas of myofibers in the diaphragm to those of the strap or tongue muscle; both ratios were much lower among the subjects who were ventilated for a longer time. The rapidity of disuse atrophy reported by Levine and colleagues militates against prolonged total rest of the diaphragm in many adults as well as infants who are at risk for hypercapnic respiratory failure.

Stephen H. Loring, M.D.

Beth Israel Deaconess Medical Center Boston, MA 02215

sloring@bidmc.harvard.edu

1. Knisely AS, Leal SM, Singer DB. Abnormalities of diaphragmatic muscle in neonates with ventilated lungs. J Pediatr 1988; 113:1074-7.

2. Schild K, Neusch C, Schönhofer B. Ventilator-induced diaphragmatic dysfunction (VIDD). Pneumologie 2008;62:33-9.

TO THE EDITOR: Levine et al. report atrophy of the diaphragm muscle due to disuse in mechanically ventilated patients. Some issues have not been addressed appropriately. Brain death is associated with alteration in the mitochondrial function of skeletal muscle. ${ }^{1}$ This alteration may induce oxidative stress and subsequent protease activation resulting in myosin loss. Information about cellular energy failure in brain-dead patients may be provided by the ratio of lactate to pyruvate in plasma. ${ }^{1}$

Atrophy was investigated solely by measuring cross-sectional area. Levine and colleagues propose that a reduction in the cross-sectional area would predict an approximately 55\% decrease in transdiaphragmatic pressure. However, a reduction in the cross-sectional area is not necessarily accompanied by reduced force generation. ${ }^{2}$ Conversely, we found that in patients with chronic obstructive pulmonary disease, the cross-sectional area of the diaphragm was not different from that of controls, but single-fiber force generation was significantly reduced (by approximately $27 \%$ ), which could be explained by a loss of myosin of approximately $29 \% .^{3}$ Thus, a prediction of reduction in force should not be based on crosssectional area, but rather on myosin content. ${ }^{3}$

Leo M.A. Heunks, M.D., Ph.D.

Richard P.N. Dekhuijzen, M.D., Ph.D.

University Medical Center Nijmegen

6500 HB Nijmegen, the Netherlands

I.heunks@ic.umcn.nl

1. Sztark F, Thicoïpé M, Lassié P, Petitjean ME, Dabadie P. Mitochondrial energy metabolism in brain-dead organ donors. Ann Transplant 2000;5:41-4.

2. Trappe S, Harber M, Creer A, et al. Single muscle fiber adaptations with marathon training. J Appl Physiol 2006;101:721-7.

3. Ottenheijm CA, Heunks LM, Sieck GC, et al. Diaphragm dysfunction in chronic obstructive pulmonary disease. Am J Respir Crit Care Med 2005;172:200-5.

TO THE EDITOR: Levine and colleagues report on the effects of controlled mechanical ventilation on atrophy of the diaphragm. Although they cite other potential contributors to atrophy of the diaphragm (e.g., systemic inflammatory response syndrome and sepsis), they do not mention critical-illness polyneuropathy and myopathy and some of its risk factors, which also may lead to muscle atrophy and dysfunction of the diaphragm in these patients. ${ }^{1}$ Critical-illness polyneuropathy and myopathy is common, with a prevalence ranging from 30 to $90 \%$, and it may develop quickly after the onset of critical illness. ${ }^{2,3}$ Glycemic control may be an important strategy to mitigate critical-illness polyneuropathy and myopathy. ${ }^{4}$ In this study, the blood glucose level was significantly higher in case patients versus controls (164 vs. $92 \mathrm{mg}$ per deciliter, $\mathrm{P}<0.001$ ), and only 4 of 14 patients received insulin infusions. We posit a potential "two-hit" hypothesis, with an interaction between muscle inactivity and the early development of subclinical critical-illness polyneuropathy and myopathy, contributing to rapid muscle atrophy in critically ill patients. This hypothesis requires investigation in future studies. Increasing awareness of critical-illness polyneuropathy and myopathy and further research on reversible risk factors and potential therapies are needed to ameliorate weakness and diaphragmatic dysfunction in critically ill patients. ${ }^{2,5}$

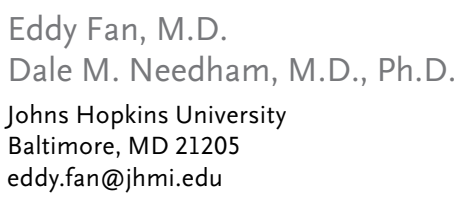


1. Kerbaul F, Brousse M, Collart F, et al. Combination of histopathological and electromyographic patterns can help to evaluate functional outcome of critically ill patients with neuromuscular weakness syndromes. Crit Care 2004;8:R358-R366.

2. Hough CL, Needham DM. The role of future longitudinal studies in ICU survivors: understanding determinants and pathophysiology of weakness and neuromuscular dysfunction. Curr Opin Crit Care 2007;13:489-96.

3. Khan J, Harrison TB, Rich MM, Moss M. Early development of critical illness myopathy and neuropathy in patients with severe sepsis. Neurology 2006;67:1421-5.

4. Stevens RD, Dowdy DW, Michaels RK, Mendez-Tellez PA, Pronovost PJ, Needham DM. Neuromuscular dysfunction acquired in critical illness: a systematic review. Intensive Care Med 2007; 33:1876-91.

5. Young GB, Hammond RR. A stronger approach to weakness in the intensive care unit. Crit Care 2004;8:416-8.

TO THE EDITOR: In the article by Levine and colleagues, controls were patients with good general health and respiratory conditions that made them candidates for surgery. In the population of case subjects, four patients had been involved in motor vehicle accidents, which is a frequent cause of transient diaphragmatic paralysis and may cause long-term diaphragmatic eventration. ${ }^{1}$ In this case, a reduction in the diaphragmatic thickness is expected, even without intubation. ${ }^{2}$ Four other patients had a history of drug or alcohol abuse. In these subjects, malnutrition is frequent and muscular impairment may involve the diaphragm.

Francesco Leo, M.D., Ph.D.

European Institute of Oncology

20141 Milan, Italy

francesco.leo@ieo.it

Lorenzo Spaggiari, M.D., Ph.D.

University of Milan School of Medicine

20141 Milan, Italy

1. Mouroux J, Venissac N, Leo F, Alifano M, Guillot F. Surgical treatment of diaphragmatic eventration using video-assisted thoracic surgery: a prospective study. Ann Thorac Surg 2005;79: 308-12.

2. Gottesman E, McCool FD. Ultrasound evaluation of the paralyzed diaphragm. Am J Respir Crit Care Med 1997;155:1570-4.

THE AUTHORS REPLY: We agree with McCool and colleagues that the case report by Ayas et al. ${ }^{1}$ provides definitive evidence that unilateral phrenicnerve stimulation can prevent atrophy - as assessed by ultrasound - in the ipsilateral hemidiaphragm. Therefore, bilateral phrenic-nerve stimulation may be useful in preventing atrophy of diaphragm fibers in patients with the combination of mechanical ventilation and diaphragmatic inactivity.
We also agree with Loring's comments about the article by Knisely et al. ${ }^{2}$ Since their infant study subjects were not brain-dead, their observations provide support for our concept that brain death per se was not playing a major role in eliciting the myofiber atrophy observed in the diaphragms of our case subjects.

With regard to the comments by Heunks and Dekhuijzen, because of the lack of clinical and histologic data in the article by Sztark et al., ${ }^{3}$ the mitochondrial dysfunction that they observed in the long peroneal muscle of their brain-dead organ-donor subjects may represent early changes associated with disuse in this limb muscle. Second, myosin concentration was not measured in our diaphragm-biopsy specimens. Therefore, in our predictions of the maximum force-generating capacity of the diaphragm, we used measured cross-sectional areas, fiber-type proportions, and assumed normal specific force for the diaphragmbiopsy specimens from both case subjects and controls. If the increased proteolysis observed in the diaphragms of the case subjects was accompanied by a decrease in myosin concentration, as compared with the diaphragms of the controls, the diaphragms of the case subjects would have shown a greater decrease in force-generating capacity than that estimated in our article (i.e., a decrease in transdiaphragmatic pressure of $>55 \%$ ).

We agree with Fan and Needham that our case subjects had hyperglycemia, which is a risk factor for both critical-illness polyneuropathy and myopathy. Therefore, these conditions should be considered as possible causes of atrophy of diaphragm myofibers. ${ }^{4}$ The absence of necrosis and hypercellularity in the diaphragm-biopsy specimens of our case subjects rules out the myopathy associated with the critical-illness polyneuropathy and myopathy syndrome. ${ }^{5}$ Since we did not obtain sections of phrenic nerve, we cannot rule out a neural contribution to the atrophy of diaphragm fibers observed in our case subjects.

Regarding the concerns of Leo and Spaggiari, we do not believe that either trauma or malnutrition played a role in eliciting the atrophy of diaphragm fibers in the case subjects because we excluded subjects with thoracoabdominal trauma from our case cohort and because these patients showed no signs of malnutrition; indeed, our data indicate that case and control subjects did not differ with respect to body-mass index. 
Sanford Levine, M.D.

Murat T. Budak, M.D., Ph.D.

Joseph B. Shrager, M.D.

University of Pennsylvania

Philadelphia, PA 19104

sdlevine@mail.med.upenn.edu

1. Ayas NT, McCool FD, Gore R, Lieberman SL, Brown R. Prevention of human diaphragm atrophy with short periods of electrical stimulation. Am J Respir Crit Care Med 1999;159: 2018-20.

2. Knisely AS, Leal SM, Singer DB. Abnormalities of diaphrag- matic muscle in neonates with ventilated lungs. J Pediatr 1988; 113:1074-7.

3. Sztark F, Thicoïpé M, Lassié P, Petitjean ME, Dabadie P. Mitochondrial energy metabolism in brain-dead organ donors. Ann Transplant 2000;5:41-4

4. Stevens RD, Dowdy DW, Michaels RK, Mendez-Tellez PA, Pronovost PJ, Needham DM. Neuromuscular dysfunction acquired in critical illness: a systematic review. Intensive Care Med 2007; 33:1876-91.

5. Kerbaul F, Brousse M, Collart F, et al. Combination of histopathological and electromyographic patterns can help to evaluate functional outcome of critically ill patients with neuromuscular weakness syndromes. Crit Care 2004;8:R358-R366.

\section{Cholesterol Gene Polymorphisms and Cardiovascular Events}

TO THE EDITOR: Kathiresan and coworkers (March 20 issue $^{1}$ find that a genotype score of nine, which was crafted from a combination of four single-nucleotide polymorphisms (SNPs) affecting high-density lipoprotein (HDL) cholesterol and five SNPs influencing low-density lipoprotein (LDL) cholesterol, is an independent risk factor for incident cardiovascular disease. Similarly, Willer et al. recently showed that 11 SNPs associated with elevated LDL levels (most of which overlap with the genes included in the article by Kathiresan et al.) increase the risk of coronary artery disease. ${ }^{2}$ In contrast, the HDL-modulating SNPs reported by Willer et al. were not associated with the risk of coronary artery disease, although the HDL genes (cholesterol ester transfer protein [CETP], hepatic lipase [LIPC], lipoprotein lipase [LPL], and ATP-binding cassette subfamily A member 1 [ABCA1]) were similar to those in the article by Kathiresan et al. By presenting a combined genotype score comprising distinct risk factors (i.e., HDL cholesterol and LDL cholesterol), Kathiresan et al. imply a predictive value of all of these SNPs; this is in clear contrast to the findings of Willer et al. Therefore, it would have been much more informative if the genotype scores for LDL and HDL genes had been reported separately, thereby allowing closer comparison with related studies.

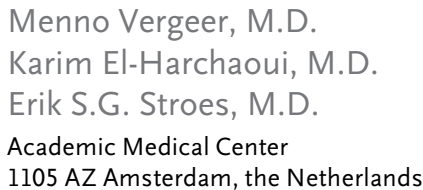

1. Kathiresan S, Melander O, Anevski D, et al. Polymorphisms associated with cholesterol and risk of cardiovascular events. N Engl J Med 2008;358:1240-9.

2. Willer CJ, Sanna S, Jackson AU, et al. Newly identified loci that influence lipid concentrations and risk of coronary artery disease. Nat Genet 2008;40:161-9.

TO THE EDITOR: Genotype scores for risk prediction are becoming increasingly popular; for instance, the use of these scores has recently been proposed to predict cardiovascular disease and prostate cancer. ${ }^{1}$ The concept relies on two major assumptions. First, polymorphisms act independently and additively on the risk of disease. Second, their effects are approximately interchangeable. The first assumption may be true, especially with regard to polymorphisms belonging to different biologic systems, but in practice it cannot be verified because current studies are underpowered to do so. The second assumption is very questionable. For example, in the study reported by Kathiresan et al., the difference in the LDL cholesterol level between homozygote genotypes was $72 \mathrm{mg}$ per deciliter for the proprotein convertase subtilisin/kexin type 9 (PCSK9) polymorphism and $3 \mathrm{mg}$ per deciliter for the LDL receptor (LDLR) polymorphism. Similarly, in terms of risk, the score assumed that it is equivalent to carry HDL-lowering or LDL-increasing alleles, despite the fact that a score based only on HDLrelated genotypes led to a hazard ratio of 1.22 , as compared with 1.11 for a score based on LDLrelated genotypes. Paradoxically, such scores take us away from the goal of individualized medicine. 